\title{
Investigating Student Preferences on Gesture-Based Interactions in a Vocabulary Game
}

\author{
Norah Sarhan, Judith Good, Kate Howland \\ University of Sussex, Brighton, United Kingdom \\ n.sarhan@sussex.ac.uk \\ j.good@sussex.ac.uk \\ k.l.howland@sussex.ac.uk
}

\begin{abstract}
There is evidence from second-language learning research that acting out vocabulary can improve learning and recall. Gesture-based language learning games have the potential to support this method of learning. A within-subjects design was used to explore student preferences for interaction styles in vocabulary games. Three types of game interaction technologies were examined: a gesture-based game in which the student points to select the vocabulary; an acting game in which the student acts out the meaning of the word to select the vocabulary; and a mouse-based game. The goal was to explore students' interaction preferences and the reasons behind their preferences as well as issues that could occur while acting out the (abstract and concrete) vocabularies. Thirty-six participants (16 males and 20 females) aged 7-9 played each of the three games in a randomly assigned order. The play sessions were video-recorded, and students participated in a short interview and survey after playing all three games. Overall, most participants enjoyed playing the acting game, suggesting that this interaction approach could be pursued in future educational game design.
\end{abstract}

Keywords: Gesture-based system; second language learning; vocabulary game; acting.

\section{Introduction}

The role of physical activity in the learning process has been widely studied, in response to theories of embodied cognition (Kosmas, loannou and Zaphiris 2018). Various different aspects of embodied learning have been studied, including the learner's use of full body movements (e.g. Johnson-Glenberg, Birchfield, Tolentino and Koziupa 2014; Lindgren, Tscholl, Wang and Johnson 2016) or gestures (Pouw et al. 2014; Roth 2001). In addition, gestures have been investigated in different educational areas such as math, physics, language learning and psychology(Kosmas, loannou and Zaphiris 2018). As discussed in the following section, a number of studies have investigated the advantages of supporting embodied learning through digital systems and examined this using empirical studies, primarily focused on learning outcomes and student's engagement and motivation( Sheu and Chen 2014). However, there has been little investigation of the use of meaningful gestures in digital learning systems, and to our knowledge, no study has focused on exploring children's interactions, whether children are interested in acting and playing with a gesture system, and their feedback concerning these systems. Therefore, the main contribution of the study presented here is the exploration of children's enjoyment and levels of comfort when engaging in meaningful gesture interactions with a system.

To explore students' opinions of acting in front of a gesture game before such a system was built, we used the Wizard of $\mathrm{Oz}$ prototyping method. This enabled us to answer the following research questions:

1. What are students' preferences for acting out vocabulary in a gesture-based English language learning game, compared with the use of pointing gestures and mouse control?

2. What design implications can be drawn from students' interactions and feedback?

\section{Background}

In the second-language learning field, several studies have examined the effect of gestures or enactments on supporting students' learning achievement and memory performance related to vocabulary acquisition. Tellier (2008) investigated the impact of using acting as part of teaching French words on young children's long-term memorisation. The results showed that children who learned by acting out the words performed significantly better on assessments. Toumpaniari et al. (2015) compared the effects of using enactments with whole-body movement and with body-part movement during a foreign language vocabulary task. They found that children who learned using whole-body movement to act out the words achieved the best learning outcomes. For learning abstract concepts, such as idioms, lonescu and llie (2016) explored whether an embodied approach in a storytelling activity could support preschool children in learning abstract expressions. Their results showed that children in the experimental group performed better when retelling the narrative sequence in order and 
when recognising new words and idioms. Schmidt et al. (2019) investigated the effect of applying meaningful and non-relevant gestures in teaching foreign language vocabulary to primary school children. They found no significant difference between the groups on the memory performance test. Another study investigated the effect of observing two different gestures (meaningful and nonmeaningful) on memory recall for children and adults. The results showed that both children and adults recalled more words when encoding them with iconic gestures (So, Sim Chen-Hui and Low Wei-Shan 2012).

Building on such promising findings, as new technologies are developed, scholars are beginning to examine the use of different digital aids, such as gesture-based learning systems, to enhance learning environments for different subjects (Kosmas, loannou and Zaphiris 2018).

In the language-learning field, Yang, Chen and Chang Jeng (2010) developed a video-capture virtual reality technology for second grade students in Taiwan that allows students to interact with the system through physical movements, they found that the system enhanced students' long-term learning. Homer et al. (2014) investigated the use of a Kinect-based storybook game to support reading comprehension for beginning readers and children. a study compared the effectiveness of three different interactions (video, gesture and 3D desktop game) for learning English to enhance learning performance for elementary students (Lan, Chen, Li and Grant 2015). Lai et al. (2018) developed a gesture-based interactive virtual learning environment to enhance language and communication skills in preschool children.

For adult learners, Chao et al. (2013) designed a system to enable students to manipulate objects in bodymotion interfaces to enhance memory performance of English phrases. Compared with a standard mousebased system, the body-motion group outperformed the mouse-based group in recall tests. Another study examined the use of Kinect to enhance memory performance in the second language learning by developing a Kinect game called SpatialEase where the adult learner responds to audio commands by moving their bodies Edge, Cheng and Whitney (2013).

In terms of motivation, Hwang et al. (2014) also used a kinaesthetic English learning system to enhance students' learning and motivation for studying a second language. Erman Yükseltürk and Serhat Altıok (2018) investigated the effects of game-based language learning with Kinect technology on students' self-efficacy and attitudes towards English, they found a significant increase of attitude and self-efficacy scores of the students in the experimental group compared to students in the control group.

The study presented here investigates children's enjoyment when engaging in meaningful gesture interactions with a system, compared with simple pointing gestures and mouse control.

\section{Methodology}

The study used a within-subjects design, which examined three versions of a vocabulary learning game. Ethical approval for this study was obtained from the University of Sussex Sciences \& Technology Cross-Schools Research Ethics Committee (SCITEC C-REC). The informed consent procedure involved written parental permission for all participants to take part in the tests and game activities, and for collection of their data. All participants also gave verbal assent in response to an assent script prior to taking part in the research activities.

\subsection{Participants and study setting}

The study was conducted in a Saudi primary school in London. The game activities and interviews took place in a school classroom. The 36 participants (16 males and 20 females) aged 7-9. All students spoke Arabic as their first language and English as their second language. 


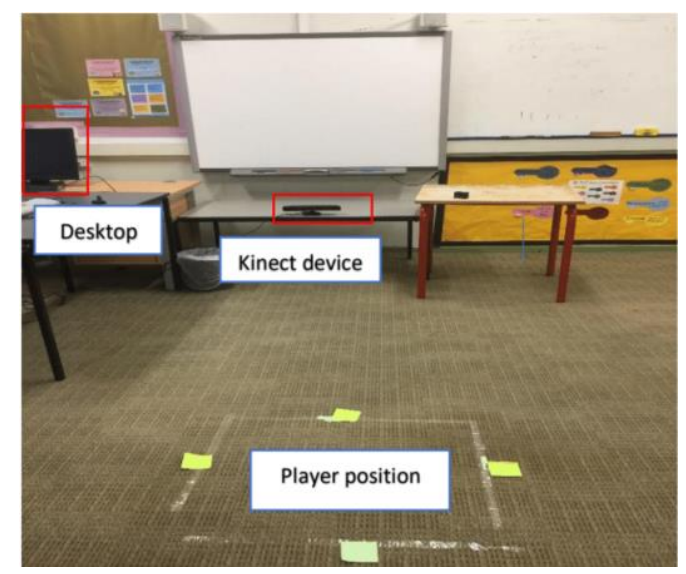

Figure 1: Study setting

\subsubsection{Materials}

The 'Marvy Learns' game from a commercial gesture gaming platform (https://kinems.com) was used. The object of the game is to categorise vocabulary words into verbs and nouns (see Figure 2). Other materials included a desktop computer, a Kinect motion capture device and a camera to record the students during the acting game. The study setting is depicted in Figure 1.

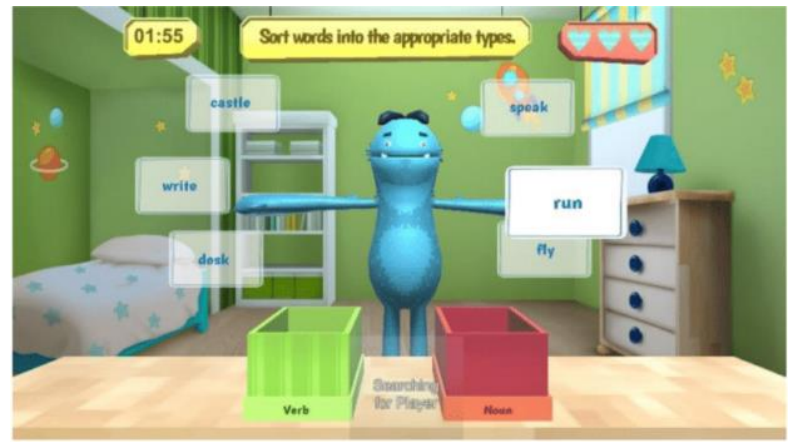

Figure 2: The 'Marvy Learns' game

\section{Game versions}

All participants played three different versions of the Marvy Learns game in a randomised order: the Kinems pointing version, an acting version and a desktop version. Kinems (also called the pointing game) is a version of the game that enables students to categorise vocabulary by interacting with a smart board using a Kinect to grab words by moving their hands (Figure 2). The acting version (also called the acting game) is identical to the Kinems pointing version except that players must act out the vocabulary word to select it. The Wizard of $\mathrm{Oz}$ method was used to implement this; participants believed that the system was able to recognise the gestures they acted out, however, the researcher was actually using the mouse to play based on the gestures the children made. The desktop version of the Marvy Learns game is identical to the others except that players are asked to use a computer mouse to select the words.

\section{Instruments}

After playing all three versions, the children were interviewed and asked about their favourite version of the game and to justify why they liked it. They were also asked to state the version they would least like to play again. The aim of this feedback was to understand their experiences and preferences regarding the game versions and identify any game design issues. In addition, a feelings survey was used to understand how the participants felt when they acted out the vocabulary words in the acting version. The participants were asked to choose one of the following emotions: 1) very unhappy, 2) unhappy, 3) neutral, 4) happy and 5) very happy. (represented as 5-point Likert scale which used a series of faces progressing from sad to happy). 


\subsubsection{Procedure}

Before the game sessions began, the researcher described the general concept of the Marvy Learns game, explaining that it is a vocabulary sorting game where six nouns and verbs need to be sorted into the correct boxes. In addition, the researcher informed the students that they would each play three different versions of the game. The order in which the game versions were played was assigned randomly.

The researcher explained each game version before the child began to play. For the pointing game, the researcher introduced the participant to the Kinect device and explained that the device could understand his/her movements and that he/she needed to stand inside the rectangle drawn on the floor and use his/her hands to select the vocabulary words on the smart board. For the acting game, the researcher introduced each participant to the Kinect device in the same way, and then introduced the acting concept of the game; that the player must act out a vocabulary word to select it. Once the word is selected, the player moves the word to the correct box using his/her hand. For each participant, the researcher began this explanation by asking, 'How do you think you can perform the word "jump"?" If the participant did not respond with a jumping action, then the researcher made a jumping gesture and asked the participant to perform a gesture for another word to ensure that the student understood what to do in the game. When the participant was ready to play the game, the researcher reminded him/her to act so that the device would understand the movements and select the appropriate word.

For the desktop game, participants use the mouse to play the game and to select the words. After they had played all three games, the researcher asked the participants the interview questions, and the participants completed the feelings survey. Each session took approximately 35 minutes.

\section{Data Collection and Analysis}

\subsubsection{Student data}

The school provided the researcher with student ages and their reading age scores (converted into months).

\subsubsection{Student preferences and feelings survey}

After the game sessions, each participant was asked the following three questions: 1) If you were allowed to play one game again, which game would you choose?; 2) Which version would you least like to play again?; and 3) When you acted out the words using your hands and body, how did you feel? (Answered by completing the feelings survey). The data were analysed as categorical variables.

\subsubsection{Participants' enactment coding}

Video recordings were made of the participants playing the acting game, and subsequently analysed. There were 31 video recordings; the remaining students could not be recorded due to technical issues with the camera. In the acting game, the children were exposed to six vocabulary words (three nouns and three verbs). The video recordings were coded according to the gestures and other actions made by the participants while they were enacting the vocabulary words.

Their acting was analysed based on Kong et al.'s (2014) gesture forms, which are iconic gestures, i.e. gestures that model the motion of an action; iconic shapes, which refers to gestures that model the shape of an object; metaphoric gestures, i.e. gestures that show pictorial content to communicate an abstract idea; and cultural gestures, meaning conventionalised signs. a category called 'incorrect gesture' was added for participants who performed an incorrect action. The data were also coded for researcher help when the researcher provided help to a participant on identifying a need, and participant asks for help when a participant asked the researcher for help.

\section{Results}

\subsubsection{Student preferences and feelings}

The Chi-square goodness-of-fit test was performed to determine whether the game types were equally preferred. The results showed that game type preference was not equally distributed across the study participants $(\chi 2(2)=7.166, p=0.027)$ and that most participants preferred the acting game (Table 1$)$. 


\begin{tabular}{||l|c|c|c|c||}
\hline \hline Age 7 & 3 & 4 & 3 & 10 \\
\hline Age 8 & 6 & 6 & 3 & 15 \\
\hline Age 9 & 2 & 9 & 0 & 11 \\
\hline Total & 11 & 19 & 6 & 36 \\
\hline
\end{tabular}

Table 1: Frequencies of participants preferring each game, by age

Table 1 shows the age distribution of participant preferences for each game. The preference for the acting game was strongest amongst the oldest children, with it being preferred by 9 of 11 nine year olds.

To investigate this further, the eta $(\eta)$ correlation ratio was calculated between the game type preference (pointing, acting, and desktop) and the participants' ages. The result indicates no association $(\eta=0.128$ ). However, an eta $(\eta)$ correlation was also run between the reading age of participants and their game preferences, and the result indicated a strong relationship $(\eta=0.713$ and $\eta 2=0.509$ ). This result suggests that the reading age of participants had a $50 \%$ effect on their game preferences. Participants preferring the acting game had greater reading age scores $(M=99.684, S D=22.78)$ compared with the pointing game $(M=85.545$, $S D=20.40)$ and the desktop game $(M=78.00, S D=19.23)$.

For the second question, a Chi-square goodness-of-fit test was performed to determine whether the game types were equally unfavoured. The minimum expected frequency was 12 . The test was statistically significant $(\chi 2(2)=10.50, p=0.005)$, with just over half of the participants choosing the desktop game as their least favourite game (Table 2 ).

\begin{tabular}{||c|c|c|c||}
\hline & POINTING GAME & ACTING GAME & DESKTOP GAME \\
\hline Favourite game & 11 & 19 & 6 \\
\hline $\begin{array}{c}\text { Least favourite } \\
\text { game }\end{array}$ & 6 & 9 & 21 \\
\hline
\end{tabular}

Table 2: Frequencies of participants' preferences

On the survey about feelings when acting out words (in the acting game), of the 36 participants, 20 chose the very happy face, 10 chose the happy face and six were neutral. A Chi-square goodness-of-fit test was performed to determine whether the Likert scale options were equally chosen. The minimum expected frequency was 12 . The test indicated that the Likert scale options were not equally distributed among the study participants $(\chi 2(2)=8.66, p=0.013)$, with just over half of the participants choosing very happy as their feeling measurement.

\subsubsection{Participants' enactment}

In the acting game, each child was assigned three nouns and three verbs. The vocabulary words were classified into two categories, abstract and concrete. Friedlander (2013) distinguished between abstract and concrete words, stating that 'abstract terms [words] refer to ideas or concepts; they have no physical referents', while 'concrete terms [words] refer to objects or events that are available to the senses' (pp. 1-2). Although, in certain contexts we can distinguish concrete vocabulary words from abstract words. It is important to note that the separating line between these terms is not sufficiently clear-cut, perhaps because they lie along a continuum that extends from most abstract to most concrete (Zhao and Macaro, 2014).

Different types of gestures were performed for the abstract and concrete words. For the abstract vocabulary, only metaphoric gestures were performed by the participants. For the metaphoric gestures, different gestures were performed for each word. In other words, each participant articulated the meanings of the words in a 
different way. For example, Children conveyed the word boy using different gestures such as playing video game, running and playing football (Figure 5).

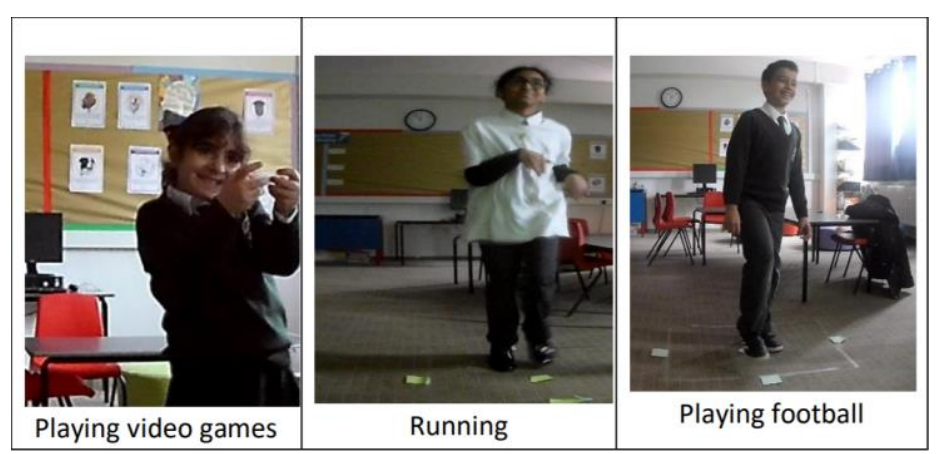

Figure 5: Participants' representations for the abstract word 'boy'

For concrete nouns, participants enacted the functionality of the object (iconic action gesture) or the participants enacted the shapes of the objects (iconic shape gesture), for example, the word 'pencil' in Figure 6. For the concrete verbs, almost all of the participants performed the same iconic action gesture for each word (for example, the word 'eat' in Figure 6); however, two verbs - 'talk' and 'speak' - were performed with a cultural gesture by moving an index finger in a round motion. This gesture is culturally known in the Middle East to indicate the action of speaking (see Figure 6).

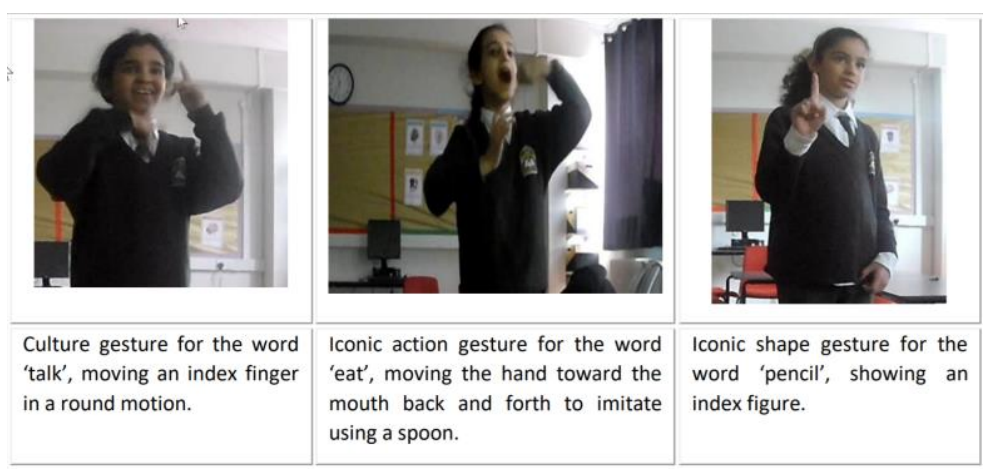

Figure 6: Participants' representations for concrete words

During the analysis of the participants' acting, various issues that arose while acting were identified. The first frequently occurring issue was difficulty acting out some vocabulary words, where participants asked for help or help was offered by the researcher. The total number of times that help was requested by the participants and offered by the researcher was counted, as shown in Table 3.

\begin{tabular}{ll|c|c|c} 
& $\begin{array}{c}\text { Number of } \\
\text { times help } \\
\text { was offered }\end{array}$ & $\begin{array}{c}\text { Number of } \\
\text { times help } \\
\text { was } \\
\text { requested }\end{array}$ & $\begin{array}{c}\text { Total number } \\
\text { of words }\end{array}$ \\
\hline $\begin{array}{l}\text { Type of } \\
\text { words }\end{array}$ & abstract & 3 & 22 & 27 \\
\hline Total & concrete & 6 & 16 & 150 \\
\hline
\end{tabular}

Table 3: Help frequencies

The second issue was unfamiliarity with vocabulary. Two participants made incorrect gestures because they did not understand the vocabulary words (the words 'talk' and 'song'). One student misinterpreted the meaning of the verb 'to watch' with the noun 'watch,' so he looked at his hand to imitate reading a watch. 


\subsubsection{Student interviews}

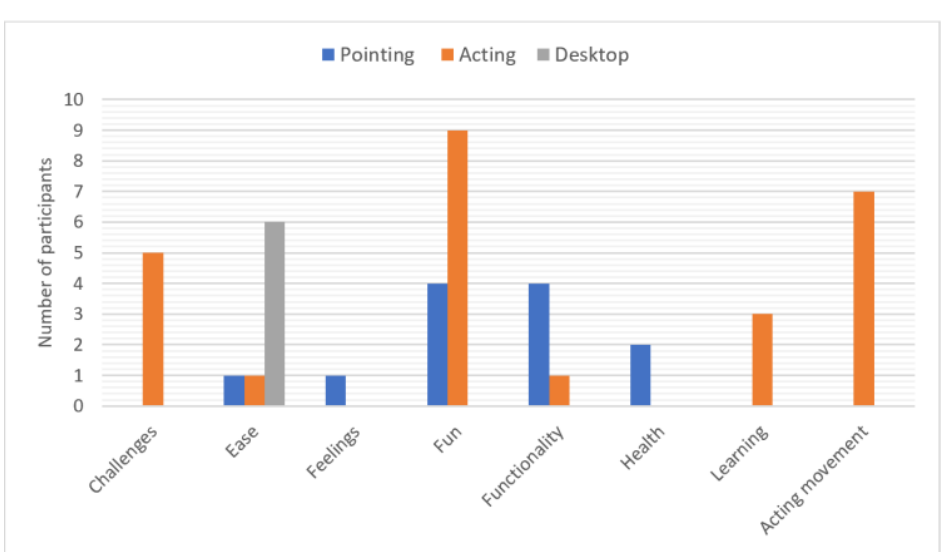

Figure 7: Categories of answers about favourite games

The interview answers were transcribed and coded using NVivo. The aim of the interviews was to understand the students' opinions of the games they rated as their most favourite and least favourite. The key themes for the version identified as their favourite games, are presented in Figure 7, with a breakdown showing which version was chosen.

For their favourite games, the highest number of statements were coded as "Fun" ( $n=13)$. For the acting game a participants says 'Sometimes it makes me laugh'. Then, the second highest category is "Ease" ( $n=8)$, the desktop game was the most frequently coded as "Ease". One participant favoured the desktop game "Because the computer was easier to use to grab the words than the other games'.

The third highest statements were "acting movement" $(n=7)$ for the acting game, One of the participants said, 'because I like [it] when I pretend to be something'. "Functionality" was mentioned for the pointing and acting games $(n=5)$, Examples of Functionality statements in the pointing game is the 3-D avatar that mimicked the player's movements, one participant said, 'It copies what I'm doing'. The "challenging" theme was only mentioned for the acting game $(n=5)$ and the "learning" category was only mentioned for the acting game $(n=3)$. The participants thought that the acting game offered challenges', whereas, for the "Learning" theme, the participants believed the acting game could help them learn to act.

The "health" and "feeling" categories were only coded for the pointing game; The participants liked the pointing game for health reasons; 'It makes my hand stronger and helps me with stretching my hand'; whereas, for the feeling category, one participant said, 'When you move your hand, you can feel that you are trying to move the word, and this helps you not [to] be nervous and relax.'

The answers relating to the version of the game the participants would least like to play again are presented in Figure 8.

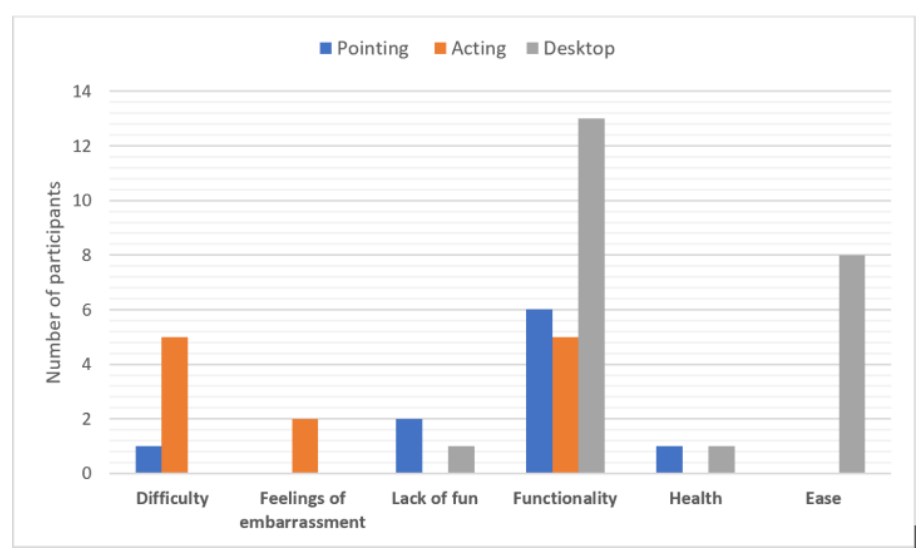

Figure 8: Categories of answers about least favourite games 
For their least favourite games, according to participant's answers, the highest number of statements were coded as "functionality" ( $n=24)$. For the desktop game, the participants mostly thought that using the mouse as a tool to select words was not interesting, this was the same for the pointing game: 'Because I just have to move the word to the box without doing anything', but for the acting game, one participant thought that having to act to play was hard 'Because it got stuck when you don't know the way to act out the word'.

The "Ease" theme was the second highest statement for the desktop game $(n=8)$, The participants did not like the desktop version because it was too easy to play. The "Difficulty" category was related to the system's response $(n=6)$. In the acting game, the participants thought that some words were difficult to act: 'I don't know how to act like a girl'.

The "lack of fun" category was only mentioned for the pointing and desktop games $(n=3)$. The "health" category was coded once in the pointing and desktop games $(n=2)$. One participant did not like the pointing game because moving their hands was uncomfortable and for the desktop game, one participant expressed a health issue: 'The closer [I was], the worse my eyes got.' Finally, the "feelings of embarrassment" category was mentioned for the acting game $(n=2)$. The participants said the acting game made them feel uncomfortable.

\section{Discussion}

\subsubsection{What are preferences for acting out vocabulary in a gesture-based English language learning game, compared with the use of pointing gestures and mouse control?}

Returning to the main question, overall, most children preferred playing the acting game more than the other two games for several reasons. According to their answers during the interviews, the majority chose the acting game because they enjoyed the activity: 'It was fun to act. Sometimes it makes me laugh'. In addition, asking children to act without mimicking someone gave them a sense of achievement ('I can learn to act'). Furthermore, the game was challenging in a positive way for some participants: 'It was challenging and fun at the same time.' It was interesting to see that they were comfortable undertaking a challenging task like acting without any instructions, and that this did not raise any feelings of embarrassment except for two participants.

The results suggested that children who preferred the acting game had a higher mean reading age than children preferring the pointing game, or the desktop game, which could be linked to ability or self-efficacy. This should be explored further in future research.

Although acting was mostly viewed positively, there were some issues related to the game during the acting itself, which were mentioned in the interviews. Sometimes, these issues resulted in children disliking the acting game.

First, choosing the word type was confusing for some of the children. Abstract words were difficult to act out without being given a model to mimic. One child said of the acting game, 'It's hard, and I found some vocabulary words difficult to act out'. There was some suggestion that words related to gender might be embarrassing to act out, such as the words 'boy' or 'girl', with one child saying 'I don't know how to act like a girl'. These issues resulted in asking for help from the researcher. Having these issues in a learning game could affect student motivation in relation to the learning experience.

Technical issues causing delays were also raised as a potential issue by the feedback. Although the game was controlled by the researcher, in some cases, the researcher did not manage to immediately select the word the first time the action was performed. Participants did not like to repeat acting more than once, as one participant said, 'Because it got stuck when you don't know the way to act out the word'.

\subsubsection{What design implications can be drawn from students' interactions and feedback?}

Based on the results of students' interviews and the acting coding, children were confident in acting out concrete vocabulary words, and they were excited to see their movement reflected by the 3-D animation.

For concrete words, such as 'sleep' and 'eat', it was easy for children to enact the word without giving them instructions. In addition, some words may confuse students. For example, the verb form of the word 'watch' has a different meaning from the noun form. It would be more effective if words that have multiple meanings are illustrated for the student. 
In terms of abstract words, such as 'play' and 'game', it was difficult for the children to create a gesture without any help. Therefore, to minimise confusion, an appropriate model on the screen of how to act out a specific word should be visible to the child. If the words are new to the children, it would be beneficial to introduce the meaning of the word either explicitly or by providing a picture to illustrate the meaning. In addition, according to our findings, designing meaningful gesture game for second language learner could be more appropriate for children with a higher reading age.

\section{Conclusion}

This paper presents children's feedback and preferences towards a digital gesture game. The study's results demonstrated that the majority of children enjoyed acting with a system (19 in total), the acting game was liked most by children aged nine ( 9 in total) and children preferring this game had a higher reading age than other children. In addition, the children were more comfortable acting out concrete words compared to abstract words.

This work is the first step in investigating the potential benefits of using enactment in digital gesture interactions for a second language-learning environment. There are some limitations to the study described in this paper. The sample size of 36 participants was relatively small. Also worth noting is that this study did not set out to investigate the factors of age group or gender, due to time and resource constraints. Consequently, future research should investigate further the role of age and reading age, and look for possible relationships between participants' gender and game preferences.

The next stage of the research involves implementing a gesture game for learning action vocabularies with a 3D animated avatar to investigate the extent to which meaningful gestural interaction can support learning vocabulary for children learning English as a second language,

\section{References}

Chao, K., Huang, H., Fang, W. and Chen, N. (2013) "Embodied Play to Learn: Exploring Kinect-Facilitated Memory Performance”, British Journal of Educational Technology, Vol. 44, No. 5, pp E151-E155.

de Koning, B.B. and Tabbers, H.K. (2013) "Gestures in Instructional Animations: A Helping Hand to Understanding Non-Human Movements?", Applied Cognitive Psychology, Vol. 27, No. 5, pp 683-689.

Edge, D., Cheng, K. Y. and Whitney, M. (2013) "SpatialEase: Learning Language through Body Motion", In Proceedings of the SIGCHI Conference on Human Factors in Computing Systems (pp 469-472).

Yukselturk, E., Altıok, S. and Başer, Z., 2018. Using game-based learning with kinect technology in foreign language education course. Journal of Educational Technology \& Society, 21(3), pp.159-173.

Gibbs, R. (2005) Embodiment and Cognitive Science, Cambridge, Cambridge University Press.

Homer, B., Kinzer, C., Plass, J., Letourneau, S., Hoffman, D., Bromley, M., Hayward, E., Turkay, S. and Kornak, Y. (2014) "Moved to Learn: The Effects of Interactivity in a Kinect-Based Literacy Game for Beginning Readers", Computers \& Education, Vol. 74, pp 37-49.

Hwang, W.Y., Shih, T.K., Yeh, S.C., Chou, K.C., Ma, Z.H. and Sommool, W. (2014) "Recognition-Based Physical Response to Facilitate EFL Learning", Journal of Educational Technology \& Society, Vol. 17, No. 4, pp 432-445.

Ionescu, T. and Ilie, A. (2016) "Language Learning in Preschool Children: An Embodied Learning Account", Early Child Development and Care, Vol. 188, No. 1, pp 4-15.

Johnson-Glenberg, M., Birchfield, D., Tolentino, L. and Koziupa, T. (2014) “Collaborative Embodied Learning in Mixed Reality Motion-Capture Environments: Two Science Studies", Journal of Educational Psychology, Vol. 106, No. 1, pp 86-104. 
Kong, A.P.H., Law, S.P., Kwan, C.C.Y., Lai, C. and Lam, V. (2015) "A Coding System with Independent Annotations of Gesture Forms and Functions during Verbal Communication: Development of a Database of Speech and Gesture (DoSaGE)", Journal of Nonverbal Behavior, Vol. 39, No. 1, pp 93-111.

Kosmas, P., loannou, A. and Zaphiris, P. (2018) "Implementing Embodied Learning in the Classroom: Effects on Children's Memory and Language Skills", Educational Media International, Vol. 56, No. 1, pp 59-74.

Lai, N., Ang, T., Por, L. and Liew, C. (2018) "Learning through Intuitive Interface: A Case Study on Preschool Learning", Computers \& Education, Vol. 126, pp 443-458.

Lan, Y., Chen, N., Li, P. and Grant, S. (2015) "Embodied Cognition and Language Learning in Virtual Environments", Educational Technology Research and Development, Vol. 63, No. 5, pp 639-644.

Lindgren, R., Tscholl, M., Wang, S. and Johnson, E. (2016) “Enhancing Learning and Engagement through Embodied Interaction within a Mixed Reality Simulation”, Computers \& Education, Vol. 95, pp 174-187.

Pouw, W., de Nooijer, J., van Gog, T., Zwaan, R. and Paas, F. (2014) "Toward a More Embedded/Extended Perspective on the Cognitive Function of Gestures," Frontiers in Psychology, Vol. 5.

Roth, W. (2001) "Gestures: Their Role in Teaching and Learning”, Review of Educational Research, Vol. 71, No. 3, pp 365-392.

Schmidt, M., Benzing, V., Wallman-Jones, A., Mavilidi, M., Lubans, D. and Paas, F. (2019) "Embodied Learning in the Classroom: Effects on Primary School Children's Attention and Foreign Language Vocabulary Learning", Psychology of Sport and Exercise, Vol. 43, pp 45-54.

Sheu, F. and Chen, N. (2014) "Taking a Signal: A Review of Gesture-Based Computing Research in Education", Computers \& Education, Vol. 78, pp 268-277.

So, W., Sim Chen-Hui, C. and Low Wei-Shan, J. (2012) "Mnemonic Effect of Iconic Gesture and Beat Gesture in Adults and Children: Is Meaning in Gesture Important for Memory Recall?", Language and Cognitive Processes, Vol. 27, No. 5, pp 665-681.

Tellier, M. (2008) "The Effect of Gestures on Second Language Memorisation by Young Children", Gestures in Language Development, Vol. 8, No. 2, pp 219-235.

Toumpaniari, K., Loyens, S., Mavilidi, M. and Paas, F. (2015) “Preschool Children's Foreign Language Vocabulary Learning by Embodying Words through Physical Activity and Gesturing", Educational Psychology Review, Vol. 27, No. 3, pp 445-456.

Yang, J., Chen, C. and Chang Jeng, M. (2010) "Integrating Video-Capture Virtual Reality Technology into a Physically Interactive Learning Environment for English Learning”, Computers \& Education, Vol. 55, No. 3, pp 1346-1356.

Yukselturk, E., Altıok, S. and Başer, Z. (2018) “Using Game-Based Learning with Kinect Technology in Foreign Language Education Course", Journal of Educational Technology \& Society, Vol. 21, No. 3, pp 159-173. 